\title{
Rol de la vegetación en el control del microclima urbano y en la adaptación a los efectos del cambio climático en un barrio de San Pedro de la Paz, Chile
}

\author{
Role of vegetation in control of urban \\ microclimate and in adaptation to effects of \\ climate change in a neighborhood located in San \\ Pedro de la Paz, Chile
}

${ }^{1}$ Javiera Pérez Jara / ${ }^{2}$ Francisco de la Barrera

RESUMEN

El cambio climático tendrá efectos sobre las áreas urbanas, por lo que es fundamental implementar medidas de adaptación y mitigación. El objetivo del estudio es evaluar los efectos del cambio climático en el microclima urbano en un barrio de San Pedro de la Paz, y el rol que ejerce la vegetación urbana en ese proceso. Se usaron datos de sensores de temperatura analizados con herramientas espaciales y Envi-met para realizar modelaciones de temperatura del aire bajo distintos escenarios de incremento de vegetación. Los resultados indican que la vegetación influye notablemente en la distribución de las temperaturas, siendo la cobertura arbórea determinante en la regulación de los efectos del microclima urbano actual y futuro, en condiciones de cambio climático. Si bien la vegetación es clave para estos propósitos, su potencial incremento depende de iniciativas de planificación y gestión a nivel local. Se evidencia, así, que el aumento de la vegetación y de su homogeneidad espacial, incide en una mayor confortabilidad microclimática. Bajo escenarios de incremento de temperaturas por el cambio climático, la vegetación urbana representa una estrategia de adaptación y mitigación recomendable de estimular.
Palabras clave

Modelación microclimática; infraestructura verde; mitigación térmica, isla de calor urbana

\section{ABSTRACT}

Climate change will have effects on urban areas, therefore it is essential to implement adaptation and mitigation measures. The objective of the study is to evaluate the effects of climate change on the urban microclimate in a neighborhood of San Pedro de la Paz, and the role that urban vegetation would play. Data from temperature sensors analyzed with spatial tools and Envi-met were used to perform models of potential air temperature under different scenarios of vegetation increase. Results indicate that vegetation notably influences the distribution of temperatures with tree cover being a determining factor in regulating the effects of the current and future urban microclimate under conditions of climate change. Although vegetation is key for these purposes, its potential increase depends on management and planning initiatives at the local level. Increasing the vegetation and homogenizing its spatial distribution will lead to more comfortable microclimatic conditions. Under climate
${ }^{1}$ Facultad de Arquitectura, Urbanismo y Geografia, Universidad de Concepción, Concepción, Chile. ORCID: https://orcid.org/0000-0001-7265-1011

2 Facultad de Arquitectura, Urbanismo y Geografia, Universidad de Concepción, Concepción, Chile ORCID: https://orcid.org/0000-0002-6854-3197 
change scenarios, urban vegetation represents a worthy strategy for adaptation and mitigation.

Keywords

Microclimatic modeling; green infrastructure; thermal mitigation, urban heat island

\section{INTRODUCCIÓN}

Las consecuencias del cambio climático en las ciudades, requieren de acciones de adaptación y mitigación mediante una planificación y gestión territorial sustentable (Kabisch et al., 2017; Santamouris, 2021). En este sentido, la concentración urbana genera excesivas emisiones de gases de efecto invernadero (GEI) que alteran la composición de la atmósfera y ocasionan desequilibrios ambientales. Estos, subyacen en una profunda degradación ambiental y energética, como son las variaciones de temperatura producto de Islas de Calor Urbanas (ICU) (Gonzáles et al., 2021) o las condiciones a las que se enfrenta la población en un corto período de tiempo en su propio entorno. Ejemplo de esto es lo que sucede con el microclima urbano (Simon y Bruse, 2020), que se encuentra determinado principalmente por los elementos del hábitat, como el comportamiento humano, la cercanía a cuerpos de agua o la vegetación, siendo esta última determinante para la adaptación a los efectos del cambio climático y control del microclima urbano (Emilsson, y Ode Sang en Kabisch et al., 2017).

En ese contexto, el objetivo de la presente investigación fue evaluar los efectos del cambio climático en el microclima urbano, así como el rol actual y potencial de la vegetación urbana en la temperatura del aire del barrio, a través de herramientas como sensores de temperatura, análisis espaciales y simulaciones microclimáticas. Se usó como área de estudio el barrio San Pedro de la Costa I, ubicado en la comuna de San Pedro de la Paz, Región del Biobío, Chile. Si bien a pesar de que, por su condición litoral, las temperaturas en San Pedro de la Paz no son extremas, sí se pueden generar diferencias importantes de temperatura producto de una configuración urbana, con más o menos vegetación. Se estima que el incremento de la vegetación, su calidad y distribución, podría ser significativo para el control del microclima urbano actual y con cambio climático, siendo fundamental como medida de adaptación y mitigación.

\section{MARCO TEÓRICO}

\section{Impacto del cambio climático y microclima urbano}

Algunos de los impactos del cambio climático que afectarían al medio urbano son: días y noches más cálidos; aumento de olas de calor, de lluvias torrenciales y de áreas afectadas por la sequía, entre otros 
(Mutizwa-Mangiza et al., 2011). De este modo, las modificaciones en las necesidades térmicas de los edificios y en las sensaciones de confort al aire libre deberán enfrentarse con transformaciones en las estrategias y en la morfología urbana, como el enverdecimiento de las ciudades (Palme et al., 2019). Por esto, es fundamental integrar una perspectiva sistémica en cuanto a la planificación urbana y su relación con otros campos de estudio a fin de abordar, en particular, cada problema de la forma más sostenible posible, mediante soluciones basadas en la naturaleza (Kabisch et al. 2017), ya que la vegetación cumple un rol fundamental en la adaptación al cambio climático (Reid y Swiderska, 2008; MMA, 2018). A escala urbana, entrega beneficios ambientales, económicos y sociales y favorece la adaptación al cambio climático regulando sus impactos negativos. Por ejemplo, reduce el consumo energético y los efectos de las ICU, tales como el estrés y la disconformidad térmica, mediante el efecto moderador de temperaturas que tienen los espacios verdes (Vásquez, 2016).

Por su parte, los procesos de urbanización reemplazan ecosistemas naturales y seminaturales por ecosistemas artificiales, lo cual afecta el balance energético de los eventos físicos y biológicos del lugar. Además, modifican las condiciones climáticas y ambientales, pues alteran los niveles de irradiación del suelo, reducen la evapotranspiración, aumentan la escorrentía superficial y disminuyen la velocidad del viento (Fernández y Martilli, 2012). Así, en el clima urbano interactúan las condiciones meteorológicas, las estructuras urbanas, y las actividades humanas (De la Barrera y Reyes-Paecke, 2020), mientras conservan las condiciones climáticas de la región en que se asienta. Esto se traduce en el aumento de la temperatura dentro de las zonas urbanas, distinguiéndose características climáticas diferentes a las de su entorno rural próximo, lo que se definió, en el año 1958, como Isla de Calor Urbana (Moreno, 1997; Salas y Herrera, 2017).

Irina Tumini (2012), señala que lo que diferencia a la ICU del microclima urbano es la escala de medición de las variaciones climáticas dentro de la ciudad. La primera, contempla un espacio más grande, donde se analizan las variaciones climáticas producidas por el conjunto urbano en el cual influye la topografía, las fuentes de agua, como ríos y lagunas, y otras grandes áreas con vegetación, como son los parques urbanos, acercándose a la definición de mesoescala (Cordero, 2014). La segunda, se acerca más a una microescala, abarcando las variaciones climáticas de sitios más reducidos, como la interacción de edificios, calles, pequeños jardines con vegetación, etc.

\section{La importancia de la vegetación urbana para el control del microclima}

Las investigaciones demuestran que las temperaturas del aire superficial varian considerablemente cuando se comparan lugares altamente construidos y con baja vegetación, con áreas urbanas que se encuentran próximas a fuentes de humedad o parques urbanos, resultando que las islas frías se encuentran en lugares con mayor vegetación (Sarricolea et al., 2008; Sarricolea y Romero, 2010). Por esto, es necesario analizar las 
infraestructuras verdes para cada escala, ya que, además de los distintos tipos, es fundamental conocer su forma, tamaño/cantidad y ubicación espacial para definir su capacidad de regular la temperatura (De la Barrera y Reyes-Paecke, 2020; Zölch et al., 2016).

Las áreas con vegetación urbana influyen en la humedad, en la infiltración del agua; controlan la erosión; regulan la temperatura, retienen contaminantes atmosféricos y, así, mitigan las ICU. Además, actúan como corredores biológicos y ofrecen recreación. Por lo tanto, indiscutiblemente, son componentes que otorgan calidad de vida y sustentabilidad urbana (Alarcón, 2009). En particular, los árboles son especialmente efectivos, no solo para la reducción de altas temperaturas (Zölch et al., 2016), sino también para la disminución de las emisiones de los vehículos y del consumo de energía; para refrigeración, en verano y calefacción, en invierno (Alarcón, 2009). Tanto los árboles, como otros elementos de la vegetación, se deben localizar estratégicamente para una mayor efectividad (Zölch et al., 2016). En Chile, los espacios verdes son aquellos que concentran más vegetación. Sin embargo, son poco efectivos porque se conciben como elementos urbanos independientes, sin conectividad con su entorno y fragmentados, limitando su funcionalidad y la conservación de la biodiversidad (Fernández y De la Barrera, 2018).

Respecto a la contribución específica de distintos elementos de la vegetación urbana, Zölch et al., (2016) resaltan la importancia del arbolado en la adaptación climática por su capacidad de generar sombra y facilitar la evapotranspiración. Además, Venter et al. (2019) lo relacionan con la salud y destacan la efectividad de los árboles para mitigar la exposición a altas temperaturas en personas especialmente sensibles al calor. Por otra parte, los techos y muros verdes han demostrado ser más importantes en la regulación de la temperatura dentro de los edificios que en el control de la temperatura urbana (De la Barrera y Reyes-Paecke, 2020). Existe un gran conjunto de otros espacios verdes, como plazas, jardines y huertos -que predominan en barrios residenciales-, que también pueden contribuir a estos propósitos, mientras se relacionen entre ellos para poder formar un sistema que alivie las temperaturas (De la Barrera y Reyes-Paecke, 2020).

\section{Herramientas geográficas para evaluar el rol de la vegetación urbana en el microclima}

La teledetección es la técnica de obtención de datos a través de imágenes satelitales y ha sido ampliamente usada para calcular temperaturas superficiales. Si bien sus resultados son consistentes, suele ser poco representativa en la microescala, por lo que, generalmente, se debe complementar con otros métodos de medición de temperatura para validar los datos.

A su vez, las mediciones con sensores de temperatura in situ son utilizadas por muchos grupos de investigación para estudiar la temperatura percibida. A modo de ejemplo, Venter et al., (2019) indican que son útiles en estudios relacionados con la salud, pues pueden 
representar la percepción de las personas. Sarricolea et al., (2008) se enfocan en las ICU nocturnas, donde complementa el uso de estaciones fijas y móviles de medición de temperatura. Finalmente, Jonghoon Park et al., (2016) concluyen que los espacios verdes son capaces de mitigar las ICU y, dependiendo del tipo de vegetación y de su distribución, pueden optimizar su efecto enfriamiento.

Por otra parte, los softwares de modelación han evolucionado y su uso permite generar simulaciones mucho más precisas, capaces de considerar más variables y mejorar la percepción de los procesos que ocurren dentro del entorno urbano. En ese contexto, ENVI-met es el de más fácil acceso y uso, y tiene como objetivo simular la interacción entre superficies construidas, vegetación y atmósfera en un entorno urbano a microescala basado en modelos de fluidodinámica y termodinámica, siendo muy útil para identificar la vegetación como ente regulador del microclima urbano (Tumini, 2012; Bruse, 2004).

\section{METODOLOGÍA}

La metodología de este trabajo se basa en la evaluación de las condiciones climáticas actuales y sus variaciones frente a diferentes escenarios donde se aumente la vegetación urbana. Se busca entender cómo esta influye en el control del microclima urbano y, a su vez, proyectarlo con condiciones de cambio climático para comprender su contribución en el control del microclima frente al aumento de las temperaturas, en el caso de estudio del barrio San Pedro de la Costa I. Para concretar el objetivo, se digitalizó la vegetación y se registraron datos de temperatura in situ. A partir de esto, se realizaron dos análisis geográficos validados en la literatura internacional, que son la interpolación espacial de temperaturas y la modelación microclimática a partir de datos de entrada registrados in situ y modificados de acuerdo a la estación meteorológica Carriel sur y las proyecciones climáticas de la Base Digital de Clima.

\section{Área de estudio}

El barrio San Pedro de la Costa I nace de un proyecto social para dar solución habitacional a familias vulnerables que vivian de forma irregular en Concepción, las que fueron reubicadas en este barrio que se emplaza desde el año 2005 en un entorno que, hasta ese año, era natural. Es un barrio que cuenta con 850 viviendas en un total de 16,5 hectáreas, donde el $45,9 \%$ corresponde a uso habitacional; $39 \%$, a calles y pasajes; $9,4 \%$, a áreas verdes (consolidadas en 2013) y un 5,4\%, a infraestructuras de equipamiento (Bastías, 2014).

\section{Descripción de los efectos del cambio climático proyectados para san pedro de la paz}

Se recogieron los datos de la Plataforma Digital de Cambio Climático, que son generales y de escala mesoclimática. Estos datos de temperatura fueron ingresados para la proyección climática de invierno y verano durante la modelación de temperaturas al año 2050. 
Digitalización de la distribución de la vegetación en el barrio

Se utilizaron imágenes satelitales de Google Earth Pro y Google Street View para espacializar el barrio y reconocer las dimensiones reales que tiene la vegetación, usando como referencia los tipos de vegetación propuestos por Fernández y De la Barrera (2018). La digitalización se basa en visitas a terreno y se realiza en ArcGIS, sobre imágenes satelitales actualizadas de enero y julio de 2020.

\section{Descripción del microclima del barrio en base a sensores de temperatura in situ.}

Se analizaron los datos de los sensores de temperatura del tipo iButton ubicados en el barrio en lugares abiertos, para medir las temperaturas en el exterior de las viviendas. Bajo criterio de contar con una representatividad territorial y espacial, así como permitir un análisis de microescala. Se instalaron, durante invierno (julio y agosto 2019), 3 sensores en espacios verdes y, durante verano (enero y febrero de 2010), 11 en antejardines y 7 en antejardines. Para analizar los datos, se estudia el día más frío de los dos meses de invierno (19/08/2019) y el día más cálido de los dos meses verano (09/02/2020) y se calcularon promedios de temperatura entre los horarios de la mañana (05:00 - 07:00), tarde (14:00 - 16:00) y atardecer (18:00 - 20:00). Se utilizó la interpolación de datos espaciales Inverse Distance Weighting (IDW) en ArcGIS, que pondera los puntos de muestreo durante el proceso, por lo que la influencia de un punto sobre otro dependerá de la distancia entre ellos. Es decir, a medida que aumenta la distancia, la influencia de este punto será menor sobre los otros. Estos resultados se contrastan con la vegetación del barrio ya digitalizada.

\section{Modelación espacial para evaluar el aporte de la vegetación}

La modelación se realiza con ENVI-met, un software de simulación térmica a microescala de entornos urbanos que se enfoca en simular la interacción entre la superficie construida, la vegetación y la atmosfera (Bruse, 2004). Este programa permite simular los efectos de la vegetación en escenarios urbanos a partir de datos climáticos de mesoescala y espaciales de microescala. Por lo tanto, es capaz de evidenciar el rol que esta tendría en el control del microclima urbano en condiciones actuales y en situaciones proyectadas, incluyendo variaciones climáticas producidas por el cambio climático. Se ingresaron, así, los parámetros climáticos de temperatura del aire y velocidad y dirección del viento que son obtenidos de la estación meteorológica Carriel Sur.

Se modeló para los mismos días y horarios utilizados en la interpolación. De estos resultados, se utiliza el horario más frío (19/08/2019 - 07:00 horas) y el más cálido (09/02/2020 - 15:00 horas). Luego se simuló una alteración en la estructura del barrio, donde la vegetación del espacio abierto pasará del 15\% presente en la actualidad, a un 50\% y $90 \%$ para visualizar la variación de las temperaturas que esto genera en el barrio. 


\section{RESULTADOS}

\section{Efectos de cambio climático proyectados para San Pedro de la}

Paz

Para la zona litoral de la comuna, se proyecta un aumento en las temperaturas de 1,9 으 entre el período de 1980 - 2010 al 2050 durante la máxima estival (enero), y de 1,3 o C durante la mínima invernal (junio). La temperatura media del período estival aumentará en promedio $1,7^{\circ} \mathrm{C}$, mientras que la del período invernal aumentará $1,3{ }^{\circ} \mathrm{C}$. Por lo tanto, la temperatura para el 2050 aumentará todos los meses del año, pero más en el período estival.

\section{Aporte de la vegetación en el control del microclima urbano}

El tipo de vegetación que predomina en el barrio es de tipo arbórea/ arbustiva y herbácea. En el barrio no hay ni techos ni muros verdes (Figura 1). La vegetación arbórea es representada principalmente por árboles de distintas especies, de tipo perenne o caducifolio que, en su mayoría, no presentan un gran follaje siendo más bien de tamaño moderado, aún en crecimiento. Las herbáceas son principalmente pasto ornamental, hierbas y malezas esporádicas. En el sector Oeste del barrio, hay 8 pasajes con nula vegetación herbácea y la vegetación arbórea tiene una distribución dispersa, si bien la mayoría de los pasajes tienen al menos un árbol o arbusto, se observan pasajes continuos donde no hay ningún tipo de vegetación de este tipo. En el sector Este, la cantidad de vegetación herbácea que se observa es mínima. La vegetación arbórea, en cambio, está mucho más presente que en el sector Oeste, en la totalidad de los pasajes se observan árboles de distintos tamaños.

Figura 1

Vegetación del barrio San Pedro de la Costa I

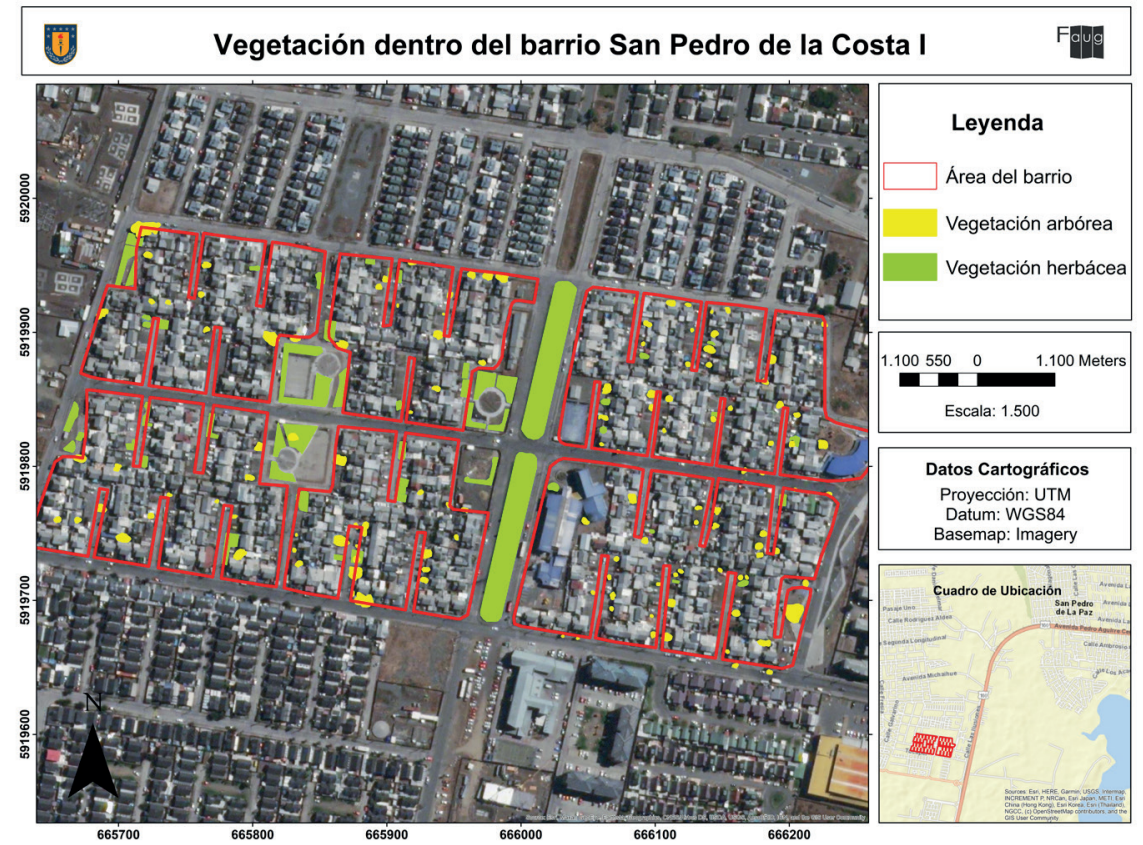

Fuente: Elaboración Propia 


\section{Microclima en San Pedro de la Costa I}

\section{Temperatura e interpolación espacial}

En invierno, la interpolación de temperaturas capturadas por sensores muestra una temperatura mínima de 8 으, en la mañana, y máxima 17,5 oC, en la tarde. Durante el momento de máxima temperatura el sector con mayor vegetación arbórea (Este) se mantiene más fresco y, junto al sensor que registró la mayor temperatura para este horario, no hay presencia de vegetación (Figura 2). En este caso, es clara la diferencia de vegetación entre ambos sectores y se observa que la vegetación arbórea cumple un rol más importante en la regulación de temperaturas.

En verano, la temperatura mínima es de 13 oC, y la máxima de 31 o.C. En general, el comportamiento térmico es irregular, con diferencias de temperaturas muy notorias durante las máximas registradas entre las 14:00 y 16:00 horas, donde se da una variación de alrededor de 8으 dentro del sector Oeste (Figura 2). Durante la tarde y el atardecer, se mantienen los mismos puntos fríos que corresponden al sector Este donde hay más vegetación arbórea, por lo que, se podría inducir que la vegetación insuficiente del sector Oeste no es relevante para regular la temperatura, mientras la del Este se encuentra levemente más regulada.

Figura 2

Interpolación de temperaturas promedio durante un día de invierno (izquierda) y de verano (derecha) en San Pedro de la Costal.

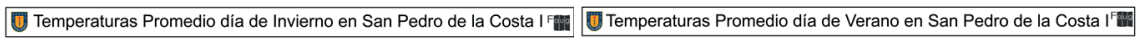
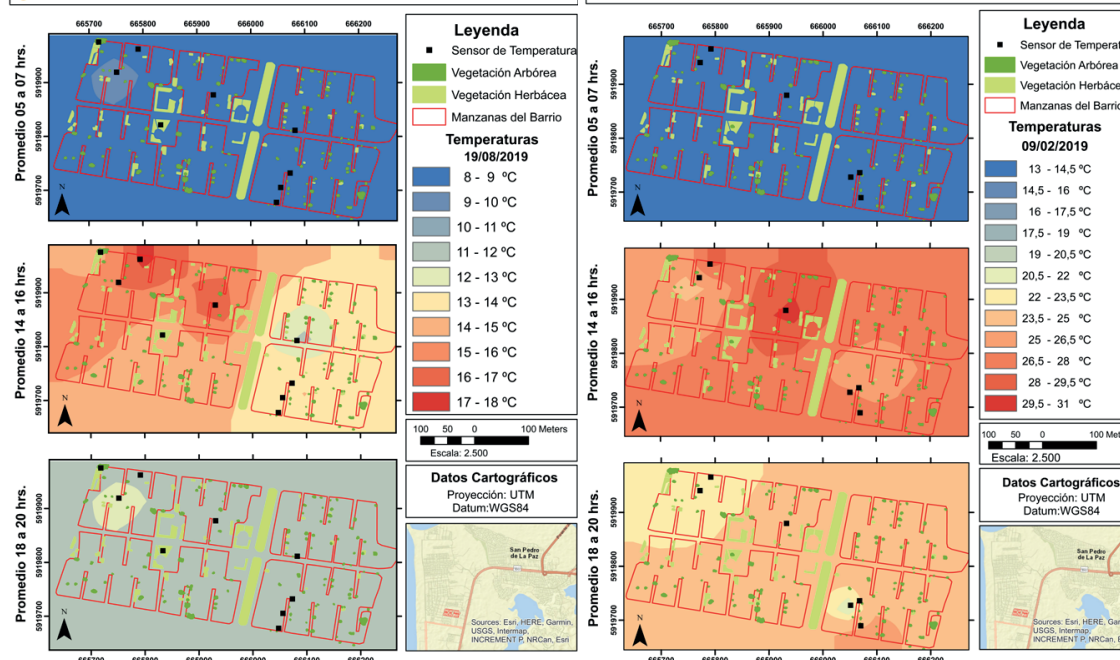

$14,5-16{ }^{\circ} \mathrm{C}$

$16-17.5^{\circ} \mathrm{C}$ $17,5-19{ }^{\circ} \mathrm{C}$
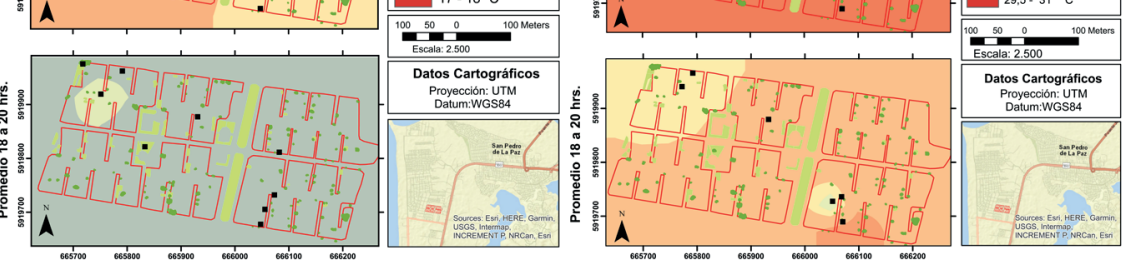

Fuente: Elaboración Propia

\section{Modelación Espacial de la Temperatura en el Barrio Usando ENVI-met}

\section{Modelación Espacial de Temperaturas}

La variación diaria en la temperatura del aire ingresada al software, fue

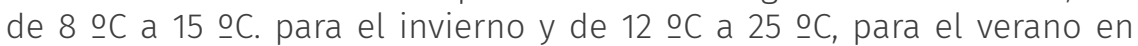
base a los datos de la estación Carriel Sur. Asimismo, el comportamiento 
de las temperaturas del aire, un día de invierno, varía entre una mínima de 10 o C , durante la mañana a 15 으 C durante la tarde, alcanzando allí el máximo de temperatura para luego disminuir a 12 으 durante el atardecer (Tabla 1). El período de la tarde es el que muestra mayor variación entre las temperaturas, se evidencian dos grandes áreas con dos rangos de temperatura que se diferencian entre sí por un grado permaneciendo entre los 13 으 y 15 으. Esto es relevante, ya que, durante la mañana y el atardecer las temperaturas son homogéneas dentro del barrio (Figura 3).

Durante el verano, el comportamiento diario de las temperaturas oscila entre 15,2 으, durante la mañana y 24,8 으, durante la tarde. Si bien esta última corresponde a la máxima del día, el barrio, durante este periodo de tiempo, se mantiene mayormente entre 22,4 oC y 23,6 으 alcanzando el máximo brevemente (Tabla 1). Hacia el atardecer, las temperaturas descienden y se mantienen entre 20 oC y 21 oC, siendo este momento del día donde la temperatura del barrio es más estable y homogénea (Figura 3).

Tabla 1

Tabla resumen de las variaciones de temperaturas durante el día en invierno y verano.

\begin{tabular}{lll}
\hline HORARIO & INVIERNO & VERANO \\
\hline $07: 00$ & Principalmente $10-1{ }^{\circ} \mathrm{C}$ & Principalmente $15,2-16,4{ }^{\circ} \mathrm{C}$ \\
\hline $15: 00$ & Principalmente $13{ }^{\circ} \mathrm{C}-1{ }^{\circ} \mathrm{C}$ & Principalmente $22,4-23,6{ }^{\circ} \mathrm{C}$ \\
\hline $19: 00$ & Principalmente $12-13{ }^{\circ} \mathrm{C}$ & Principalmente $20-21{ }^{\circ} \mathrm{C}$ \\
\hline
\end{tabular}

Fuente: Elaboración Propia 
Figura 3

Modelación de las temperaturas del aire en invierno y verano.
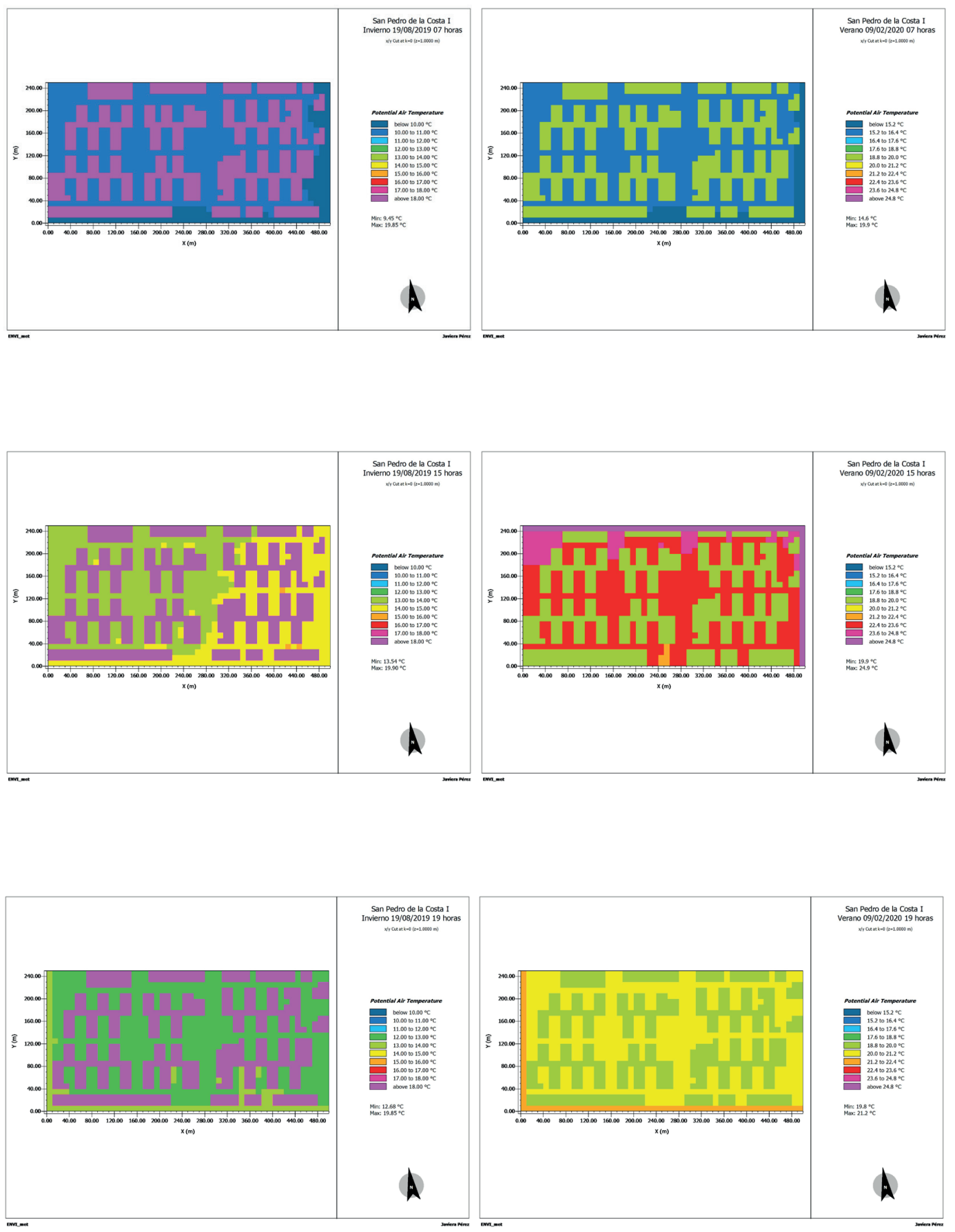

Fuente: Elaboración Propia 


\section{Evaluación del rol de la vegetación en la regulación del microclima urbano a través de una simulación del incremento en su cobertura vegetal en el barrio}

Con la modelación, las temperaturas a las 7am en invierno oscilan entre los 9,4 으 y 19,8 으 con 15\% de vegetación, aunque, mayoritariamente, bajo 11,5 ${ }^{\circ}$ C. Esto se conserva en escenarios de aumento al 50 y al 90\% de vegetación. Con la vegetación actual, existe un $37 \%$ de pixeles con temperaturas bajo 10,5 우; con vegetación al 50\%, hay 36\% y al 90\%, hay 35\% (Tabla 2). Así, para un día frío de invierno se puede decir que, al aumentar la vegetación, aumenta también la temperatura, aunque muy sutilmente (Figura 4).

Tabla 2

Porcentaje de pixeles por rango de temperatura para invierno.

\begin{tabular}{llll} 
& Cobertura & & \\
\hline Rangos de ${ }^{0}$ & $\begin{array}{l}\text { Vegetación actual: } \\
15 \% \text { de cobertura }\end{array}$ & $\begin{array}{l}\text { Simulación de } \\
50 \% \text { de cobertura }\end{array}$ & $\begin{array}{l}\text { Simulación de } \\
90 \% \text { de cobertura }\end{array}$ \\
\hline Bajo $10,5^{\circ} \mathrm{C}$ & $36,88 \%$ & $36,08 \%$ & $35,36 \%$ \\
\hline $10,5^{\circ} \mathrm{C}-11,5^{\circ} \mathrm{C}$ & $19,36 \%$ & $20,16 \%$ & $20,88 \%$ \\
\hline
\end{tabular}

Fuente: Elaboración Propia

En cambio, en verano -de acuerdo a la modelación- la temperatura oscila entre 19,9 드 y 24,9 으 durante la tarde. Se evidencia que entre 22 으 y 22,5 으 en condiciones normales de vegetación se encuentran el $11 \%$ del barrio, lo que disminuye a $8 \%$ para vegetación al $50 \%$ y aumenta a $20 \%$ con una vegetación al $90 \%$, por efecto de una mayor regulación de las altas temperaturas (Tabla 3). Los rangos entre 23,5 oC y 24 oC y el de

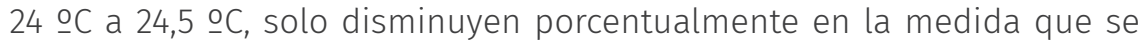
aumenta la vegetación pasando de 4,08\% a 2,96\% (rojo) y de 2,8\% a 1,6\% (rosa) entre la vegetación actual y al $90 \%$ respectivamente (Figura 4).

Tabla 3

Porcentaje de pixeles por rango de temperatura para verano

\begin{tabular}{llll} 
& Cobertura & \\
\hline Rangos de ${ }^{0}$ & $\begin{array}{l}\text { Vegetación actual: } \\
15 \% \text { de cobertura }\end{array}$ & $\begin{array}{l}\text { Simulación de } \\
50 \% \text { de cobertura }\end{array}$ & $\begin{array}{l}\text { Simulación de } \\
90 \% \text { de cobertura }\end{array}$ \\
\hline $22{ }^{\circ} \mathrm{C}-22,5^{\circ} \mathrm{C}$ & $10,96 \%$ & $8,32 \%$ & $20,48 \%$ \\
\hline $22,5{ }^{\circ} \mathrm{C}-23{ }^{\circ} \mathrm{C}$ & $25,2 \%$ & $26,96 \%$ & $19,2 \%$ \\
\hline $23{ }^{\circ} \mathrm{C}-23,5^{\circ} \mathrm{C}$ & $7,12 \%$ & $9,04 \%$ & $6,16 \%$ \\
\hline $23,5^{\circ} \mathrm{C}-24^{\circ} \mathrm{C}$ & $4,08 \%$ & $4,08 \%$ & $2,96 \%$ \\
\hline $24{ }^{\circ} \mathrm{C}-24,5^{\circ} \mathrm{C}$ & $2,8 \%$ & $2 \%$ & $1,6 \%$ \\
\hline Sobre $24,5^{\circ} \mathrm{C}$ & $6,16 \%$ & $5,92 \%$ & $5,96 \%$ \\
\hline
\end{tabular}

Fuente: Elaboración Propia 
Figura 4

Modelación de temperaturas con aumento en la cobertura vegetal.
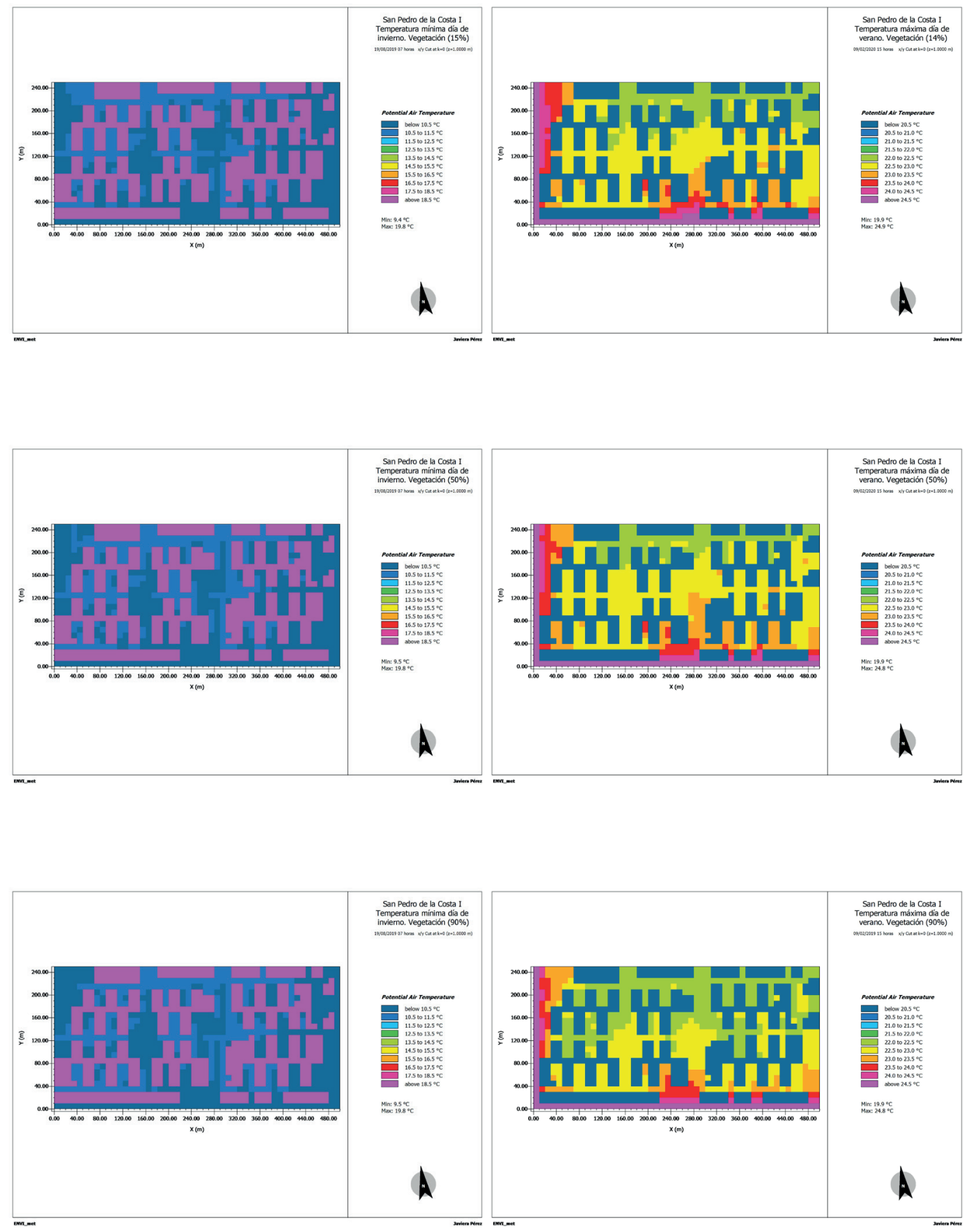

Fuente: Elaboración Propia 
Considerando los efectos del cambio climático en un incremento de $2^{\circ} \mathrm{C}$ de la temperatura, en la modelación de invierno las temperaturas son: mínima 11,4 oㄷ y máxima 19,8 으. Esto es mayor que las temperaturas introducidas y las esperadas para el 2050. No se observan variaciones importantes entre las modelaciones, pero los cálculos indican que con vegetación actual el 29\% de la superficie tendrá una temperatura del aire inferior a 12, 2 으 mientras que con 50\% de vegetación esta aumenta a $31 \%$ y con $90 \%$ de vegetación a 33\% (Tabla 4). Esto deja al descubierto que las temperaturas del aire en el barrio disminuirian mientras se aumente la vegetación. A pesar que las temperaturas aumenten 2 o- C aprox., la vegetación es capaz de regular esta situación disminuyendo las temperaturas para que se mantengan lo más parecidas a las condiciones actuales a pesar de su aumento (Figura 5).

Tabla 4

Porcentaje de pixeles por rango de temperatura para invierno con variación climática.

\begin{tabular}{llll} 
& Cobertura & \\
\hline Rangos de $T^{0}$ & $\begin{array}{l}\text { Vegetación actual: } \\
15 \% \text { de cobertura }\end{array}$ & $\begin{array}{l}\text { Simulación de } \\
50 \% \text { de cobertura }\end{array}$ & $\begin{array}{l}\text { Simulación de } \\
90 \% \text { de cobertura }\end{array}$ \\
\hline Bajo $12,5^{\circ} \mathrm{C}$ & $38,96 \%$ & $30,72 \%$ & $32,64 \%$ \\
\hline $12,2{ }^{\circ} \mathrm{C}-13^{\circ} \mathrm{C}$ & $27,36 \%$ & $25,6 \%$ & $23,68 \%$ \\
\hline
\end{tabular}

Fuente: Elaboración Propia

Por otra parte, en la modelación con cambio climático en verano $\left(+2^{\circ} \mathrm{C}\right)$ la temperatura oscila entre 19,9 으 y 27,8 으, por encima de la proyección de la "Base digital del clima del MMA". Se observa una leve variación entre la simulación con vegetación actual y al 50\%, sin embargo, al 90\% se evidencia un aumento en la superficie con bajas temperatura. En el resto de los rangos las variaciones no son explicitas. El rango de mayor temperatura ( 27 oC - 28 o C) disminuye de $8 \%$ a $7 \%$ al aumentar al máximo la vegetación. Lo mismo sucede con el rango entre 26 으 y 27으 pasando de $8 \%$ a $7 \%$ entre $15 \%$ y $50 \%$ de vegetación, y luego a $5 \%$ para el $90 \%$ (Tabla 5). Ya que estos rangos disminuyeron en superficie, los rangos de menor temperatura aumentan, es decir, las máximas temperaturas se disipan al aumentar la vegetación (Figura 5).

Tabla 5

Porcentaje de pixeles por rango de temperatura para verano con variación climática.

\begin{tabular}{llll}
\hline $\begin{array}{l}\text { Cobertura } \\
\text { Rangos de } \mathrm{T}^{0}\end{array}$ & $\begin{array}{l}\text { Vegetación actual: } \\
15 \% \text { de cobertura }\end{array}$ & $\begin{array}{l}\text { Simulación de } \\
50 \% \text { de cobertura }\end{array}$ & $\begin{array}{l}\text { Simulación de } \\
90 \% \text { de cobertura }\end{array}$ \\
\hline $26{ }^{\circ} \mathrm{C}-27{ }^{\circ} \mathrm{C}$ & $4 \%$ & $3,44 \%$ & $13,2 \%$ \\
\hline $27^{\circ} \mathrm{C}-28{ }^{\circ} \mathrm{C}$ & $37,04 \%$ & $39,04 \%$ & $31,52 \%$ \\
\hline $26{ }^{\circ} \mathrm{C}-27^{\circ} \mathrm{C}$ & $7,28 \%$ & $6,96 \%$ & $5,04 \%$ \\
\hline $27{ }^{\circ} \mathrm{C}-28{ }^{\circ} \mathrm{C}$ & $8 \%$ & $6,88 \%$ & $6,56 \%$ \\
\hline
\end{tabular}


Figura 5

Modelación de temperaturas con aumento en la cobertura vegetal y con proyección climática.
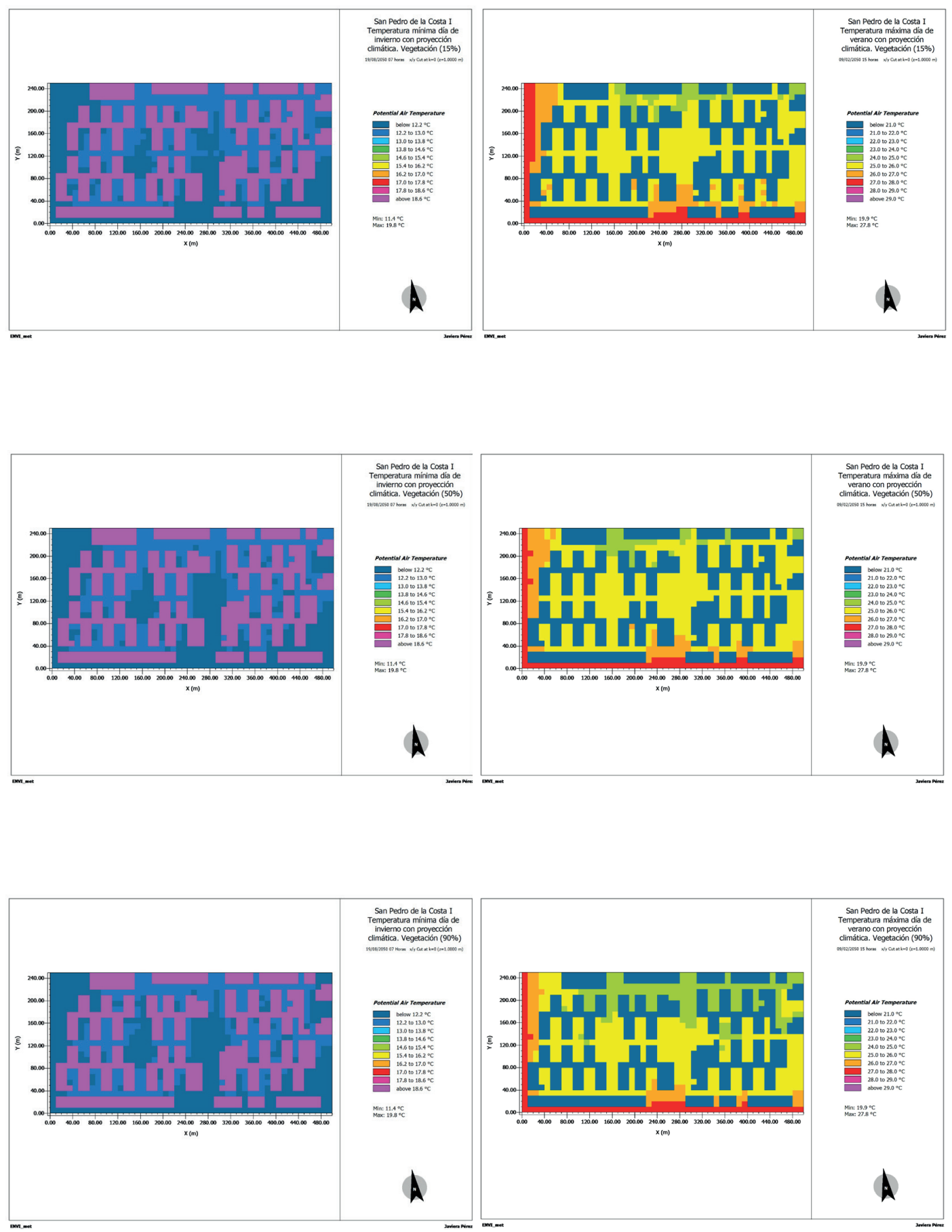

Fuente: Elaboración Propia 


\section{DISCUSIÓN}

El incremento de la vegetación es importante para la regulación microclimática, ya que se ha demostrado que, al aumentarla considerablemente, se regula la temperatura. Para que esta variación sea significativa, hay que aumentar de $15 \%$ a $90 \%$ la cantidad de vegetación en los espacios abiertos del barrio, lo que en la realidad se dificulta, por competencias en usos del espacio, recursos otros intereses de sus habitantes, que en ocasiones prefieren pavimentar para habilitar estacionamientos o preferir usos sin vegetación. En el barrio analizado solo hay arbolado; no hay ni techos ni muros verdes. Sin embargo, Bartesaghi-Koc et al., (2017); Zölch et al., (2016) y Venter et al., (2019) son algunos de los autores que han concluido que son los árboles son los que más aportan en la regulación microclimática, a escala de suelo y en la adaptación.

Si bien alcanzar un 90\% de cobertura vegetal en espacios abiertos de un barrio parece una meta difícil de alcanzar, esta puede estar dada por copas de árboles, habiendo otros usos bajo ellos o por coberturas herbáceas que puedan servir por ejemplo de estacionamientos. Asimismo, a esto debe sumarse la posibilidad de desarrollar techos verdes. Se debe tener en cuenta que los altos grados de cobertura vegetal solo son posibles en climas de alta humedad, dado que en sitios áridos o semiáridos el crecimiento de la vegetación podría tener elevados requerimientos hídricos que hagan este tipo de estrategias insostenibles. Al respecto, los grados máximos de cobertura vegetal deben definirse según el contexto geográfico, tomando en consideración tanto el clima como la cobertura vegetal natural de los alrededores naturales (De la Barrera y Henríquez, 2017).

No solo es importante que existan espacios con vegetación en los barrios, sino que las características de la vegetación se enfoquen en ser realmente eficientes para sus necesidades. En este sentido, el programa ENVI-met tiene una completa base de datos de especies de vegetación, incluyendo fenología, altura y formas de follaje/copa en el caso de los árboles, pero también arbustos o herbáceas, lo que permite obtener resultados más precisos. En la simulación se ubicaron árboles de hasta 15 metros con distintos follajes bien distribuidos y la vegetación herbácea en forma de pasto. Los resultados de la modelación espacial en ENVI-met confirman que la vegetación es capaz de regular la temperatura del aire dentro del microclima urbano y, mientras más vegetación exista, menor serán las islas de calor atmosféricas en el barrio. Sobre todo, en días de verano, cuando las temperaturas sean más altas. En cualquier caso, la temperatura será, al menos, 1,5 ㅇ más alta en el 2050 y, aumentar la vegetación del barrio con arbolado y estructuras verdes al 50\% o más, permitiría regular el microclima.

\section{CONCLUSIONES}

Las condiciones estructurales del barrio San Pedro de la Costa I determinan el comportamiento su microclima urbano. En todos los casos, incrementar la vegetación contribuye a regular las temperaturas del 
barrio, principalmente durante la tarde. Con las proyecciones climáticas al 2050 -y que implican un aumento de 2 으-, las máximas temperaturas son amortiguadas en un escenario donde la vegetación sea de 90\%, mitigando eventos extremos de calor. Lo mismo sucede en invierno, donde la vegetación permite mitigar las temperaturas mínimas.

La evidencia de los beneficios de contar con una mayor cobertura de vegetación en barrios con las características del caso de estudio, puede ser incorporada para la elaboración de instrumentos de gestión locales, tanto para el financiamiento de iniciativas como para inducir una planificación territorial sensible al clima. Al respecto, el desarrollo de ordenanzas para incentivar la incorporación de vegetación, en general, y de árboles, en particular, en los espacios abiertos disponibles debería ser una prioridad en el corto plazo. Asimismo, para el largo plazo, el desarrollo de instrumentos de planificación debería considerar los riesgos asociados al cambio climático y asegurar la disponibilidad de espacios para incorporar vegetación.

\section{REFERENCIAS BIBLIOGRÁFICAS}

Alarcón, A. (2009). Análisis de la relación entre morfología y microclima urbano y el material particulado en las comunas de Santiago Centro y Las Condes. Tesis de Magíster. Universidad de Chile. http://mgpa.forestaluchile.cl/Tesis/Alarc\%C3\%B3n,\%20Alicia.pdf

Bartesaghi Koc, C., Osmond, P. y Peters, A. (2017). Towards a comprehensive green infrastructure typology: a systematic review of approaches, methods and typologies. Urban Ecosyst 20, 15-35. https://doi. org/10.1007/s11252-016-0578-5

Base Digital del Clima. (2017). http://basedigitaldelclima.mma.gob.cl/

Bastías, C. (2014). Espacio Público y seguridad. Análisis espacial del diseño urbano en el sector de San Pedro de la Costa y sus representaciones en la percepción de seguridad y calidad de vida de sus habitantes. Seminario de título para optar al grado de arquitecto. Universidad de Concepción.

Bruse, M. (2004). ENVI-met 3.0: Updated Model Overview. www.envi-met.com

De la Barrera, F., y Reyes-Paecke, S. (2020). Green infrastructures to mitigate extreme temperatures in cities. En: Palme M y ASalvati (eds). Urban Microclimate Modelling for Comfort and Energy Studies.

De la Barrera, F., y Henríquez, C. (2017). Vegetation cover change in growing urban agglomerations in Chile. Ecological Indicators, 81, 265-273.

Fernandez, F. y Martilli, A. (2012). El Clima urbano: aspectos generales y su aplicación en el área de Madrid. Índice: Revista de Estadística y Sociedad, (50), 21-24. 
Fernández, I. y De la Barrera, F. (2018). Biodiversidad urbana, servicios ecosistémicos y planificación ecológica: un enfoque desde la ecología del paisaje. Capítulo 4. Pp.113-146.

Kabisch, N., Korn, H., Stadler, J. y Bonn, A. (2017). Nature-based Solutions to Climate Change Adaptation in Urban Areas. Linkages between Science, Policy and Practice. https://doi.org/10.1007/978-3-31956091-5

Ministerio del Medio Ambiente (MMA). (2018). Plan de Adaptación al CC en Ciudades 2018-2022. (En proceso de edición y diseño).

Moreno, M. (1997). Una propuesta de terminología castellana en climatología urbana. Investigaciones geográficas. 17, 89-97. https://www.investigacionesgeograficas.com/article/view/1997n17-una-propuesta-de-terminologia-castellana-en-climatologiaurbana

Mutizwa-Mangiza, N., Arimah, B., Jensen, I., Abera Yemeru, E., y Kinyanjui. M. (2011). Las ciudades y el cambio climático: Orientaciones para políticas. Informe mundial sobre asentamientos humanos 2011. Programa de las Naciones Unidas para los Asentamientos Humanos. Resumen Ejecutivo. https://www.zaragoza.es/ contenidos/medioambiente/onu/538-spa-ed2011-sum.pdf

Palme, M., Clemente, C., Cellurale, M., Carrasco C. y Salvati, A. (2019) Estrategias de mitigación de la intensidad de las islas de calor urbano en climas mediterráneos: estudios de simulación en Roma (Italia) y Valparaíso (Chile). IOP Conf. Ser.: Earth Environ. Sci. 323 012025. https://doi.org/10.1088/1755-1315/323/1/012025

Park, J., Kim, Jun-Hyun., Kun Lee, D., Yeon, C., y Gyu Jeong, S. (2016) The influence of small green space type and structure at the Street level on urban heat island mitigation. Urban Forestry \& Urban Greening 21. Pp. 203-2012. 10.1016 / j.ufug.2016.12.005

Reid, H., y Swiderska, K. (2008). Biodiversidad, cambio climático y pobreza: una exploración de los vínculos. International Institute for Environment and Development.

Salas, M., y Herrera, L. (2017). La vegetación como sistema de control para las islas de calor urbano en Ciudad Juárez, Chihuahua. Revista Hábitat Sustentable, 7(1), 14-23. http://revistas.ubiobio.cl/index. php/RHS/article/view/2737/2310

Santamouris, M. (2021). Present and Future Challenges and Opportunities in the Built Environment. En: Chiesa G. (eds) Bioclimatic Approaches in Urban and Building Design. Pp. 111-116. PoliTO Springer Series. https://doi.org/10.1007/978-3-030-59328-5_5 
Sarricolea, P., Aliste, E. y Escobedo, C. (2008). Análisis de la máxima intensidad de la isla de calor urbana nocturna de la ciudad de Rancagua (Chile) y sus factores explicativos. Revista de Climatología, 8, 71-84. https://www.researchgate.net/ publication/26576059

Sarricolea, P. y Romero, H. (2010). Análisis de los factores condicionantes sobre las temperaturas de emisión superficial en el área metropolitana de Valparaíso, Chile. Arquitectura, Ciudad y Entorno (ACE). 79-96. http:// repositorio.uchile.cl/handle/2250/117841

Simon, H. y Bruse, M. (2020) Modelling of Microclimates. En: Peuportier, B., Leurent, F., y Roger-Estrade, J. (Eds.). Eco-Design of Buildings and Infrastructure: Developments in the Period 2016-2020 (1st ed.). CRC Press. https://doi.org/10.1201/9781003095071

Tumini, I. (2012). El microclima urbano en los espacios abiertos. Estudio de casos en Madrid. Tesis para optar al grado de Doctor Arquitecto-Ingeniero. Universidad Politécnica de Madrid. http:// oa.upm.es/14893/1/IRINA_TUMINI.pdf

Vásquez, A. (2016). Infraestructura verde, servicios ecosistémicos y sus aportes para enfrentar el cambio climático en ciudades: el caso del corredor ribereño del río Mapocho en Santiago de Chile. Revista de Geografía Norte Grande, (63), 63-86. https://dx.doi. org/10.4067/S0718-34022016000100005

Venter, Z., Hjertager Krog, N. y Barton, D. (2019). Linking green infrastructure to urban heat and human health risk mitigation in Oslo, Norway. Science of the Total Environment, 709. https://doi.org/10.1016/j. scitotenv.2019.136193

Zölch, T., Maderspachera, J., Wamslerb, C. y Pauleitc, S. (2016). Using green infrastructure for urban climate-proofing: An evaluation of heat mitigation measures at the micro-scale. Urban Forestry \& Urban Greening, 20, 305-316. https://doi.org/10.1016/j.ufug.2016.09.011 